\section{The effects of a novel stimulus introduced into a DRL schedule} at two temporal placements*

\author{
JOYCE J. CONTRUCCI, DAVID HOTHERSALL, and DELOS D. WICKENS \\ The Ohio State University, Columbus, Ohio 43212
}

A potentially disinhibiting novel stimulus was introduced early or late into a 20-sec DRL interval after Ss had attained criterion performance on this schedule. Evidence was found for disinhibition, the inhibition of inhibition, of the leverpressing response. The effect decreased over stimulus presentations within any one test session and likewise decreased over test days. These results lend support to a traditional interpretation of inhibition and the disinhibition phenomenon rather than a mediating response explanation.

After a long period of neglect, the concept of inhibition has recently been reinstated in the psychological literature. Staddon (1969) pointed out that this reinstatement resulted from a number of developments: in sensory physiology the anatomical isolation of inhibitory neural units (Hartline, 1949); experimental demonstrations of inhibition in operant behavior (Brown \& Jenkins, 1967); and the mathematical and computational techniques for dealing with inhibition as a property of complex systems. In a related development there have been a number of recent reports of "inhibition of inhibition," or disinhibition, in operant conditioning experiments. These disinhibitory effects appear similar to those first reported by Pavlov (1927) in classical conditioning situations.

With the introduction of a novel stimulus, Ferster \& Skinner (1957) demonstrated the disappearance of the FI-scallop pattern of responding of one pigeon working on a FI 20-min schedule of reinforcement. Similar results have been reported in more recent experiments.

Flanagan \& Webb (1964) presented auditory stimuli to rats responding on a FI 1-min schedule and found disruption of the FI scallop. Hinrichs (1968) used pigeons as $S s$ and reported that changes in key color led to the disappearance of FI scallops and a moderate rate of responding throughout the fixed schedule. Singh \& Wickens (1968) trained rats on a FI 3-min schedule and found that the introduction of a novel stimulus produced marked disruption of the FI scallops on the first day of testing.

The present experiment was designed to investigate the effects of a novel stimulus presented at two temporal placements in a differential reinforcement of low-rate responding

* This research was supported in part by National Institute of Mental Health Training Grant MH08526.
(DRL) schedule of reinforcement. With such a schedule, only the response separated from the immediately preceding one by a specified period of time is reinforced, and so the $S$ is required to respond at a low rate in order to obtain reinforcements consistently. Since some type of inhibitory process might be assumed to underlie such low-rate responding, the disinhibiting effects of a novel stimulus can be investigated.

\section{SUBJECTS}

Eighteen approximately 100-day-old Sprague-Dawley rats served as Ss. The rats were maintained on ad lib water throughout the experiment, and were tested under 22-h food deprivation.

\section{APPARATUS}

Three conventional operant conditioning boxes housed in sound-attenuating ventilated chambers were used. A continuous white noise piped into the experimental room served to mask extraneous stimuli. Noyes food pellets of $45 \mathrm{mg}$ were used as reinforcers. Electromechanical programming equipment, counters, timers, and cumulative recorders were kept in an adjoining room. A buzzer the potentially disinhibiting stimulus, was mounted outside a wall of each box. When activated, the buzzer increased the noise level in the chamber to $68 \mathrm{~dB}$.

\section{PROCEDURE}

Following initial handling and taming, the rats were placed on the food-deprivation schedule for 5 days and were then individually magazine trained and shaped to leverpress in one of the operant conditioning boxes. Following shaping, all rats were placed on a CRF schedule for 75 reinforcements. On the following day, 10 reinforcements were earned on a DRL 10 -sec schedule, 10 reinforcements on a DRL 15-sec schedule, and the rats were then run for the remainder of the $60-\mathrm{min}$ session on a DRL 20-sec schedule.
During the rest of the experiment, all Ss were run for $1 \mathrm{~h}$ per day on the D R L 20 -s e c s chedule of reinforcement. Each daily $1-h$ session was divided into two 30 -min periods, and for each of these periods an efficiency ratio of reinforcements to responses was calculated (Brady \& Conrad, 1960). The DRL training was continued until attainment of a criterion of 3 successive days on which the efficiency ratio for the second $30 \mathrm{~min}$ of the $1-\mathrm{h}$ session was .50 or greater.

On the 3 days subsequent to attainment of this criterion, the following disinhibition test procedure was instituted during the second $30 \mathrm{~min}$ of the 1-h session. The rat was allowed to earn 10 reinforcements, with the number of "error responses" between each of these reinforcements being recorded. An error response was any response which did not produce a reinforcement, i.e., a response emitted before the end of the 20-sec DRL interval. The buzzer was presented for the first time between the 10th and 11 th reinforcements. Ss were then allowed to earn nine more reinforcements, and the stimulus was presented again between the 20 th and the 21st reinforcements; following 9 more reinforcements the stimulus was presented between the 30 th and the $31 \mathrm{st}$ reinforcements, and again between the 40th and the 41st reinforcements. For one group of Ss the stimulus onset was $6 \mathrm{sec}$ into these designated intervals; for the other group its onset was 12 sec into these intervals. Nine rats were assigned randomly to each of the two groups. For both groups the stimulus duration was 4 sec. This disinhibition test procedure was followed for 3 days. Comparisons were made of the number of error responses in the periods before, during, and following each stimulus presentation. Evidence of disinhibition would be found in an increased number of error responses in periods when the potentially disinhibiting stimulus was presented.

\section{RESULTS}

The group mean number of hours to attainment of the criterion DRL performance was $19.56 \mathrm{~h}$ for the group stimulated from the 6 th to the 10 th sec and $20.11 \mathrm{~h}$ for the group stimulated from the 12th to the 16 th sec. This difference was not statistically significant $(t=.12$, df $=16$ ). Figure 1 shows the number of error responses made before, during, and after each of the four stimulus presentations on each of the 3 test days for both the "early" and the "late" stimulus presentation groups. 


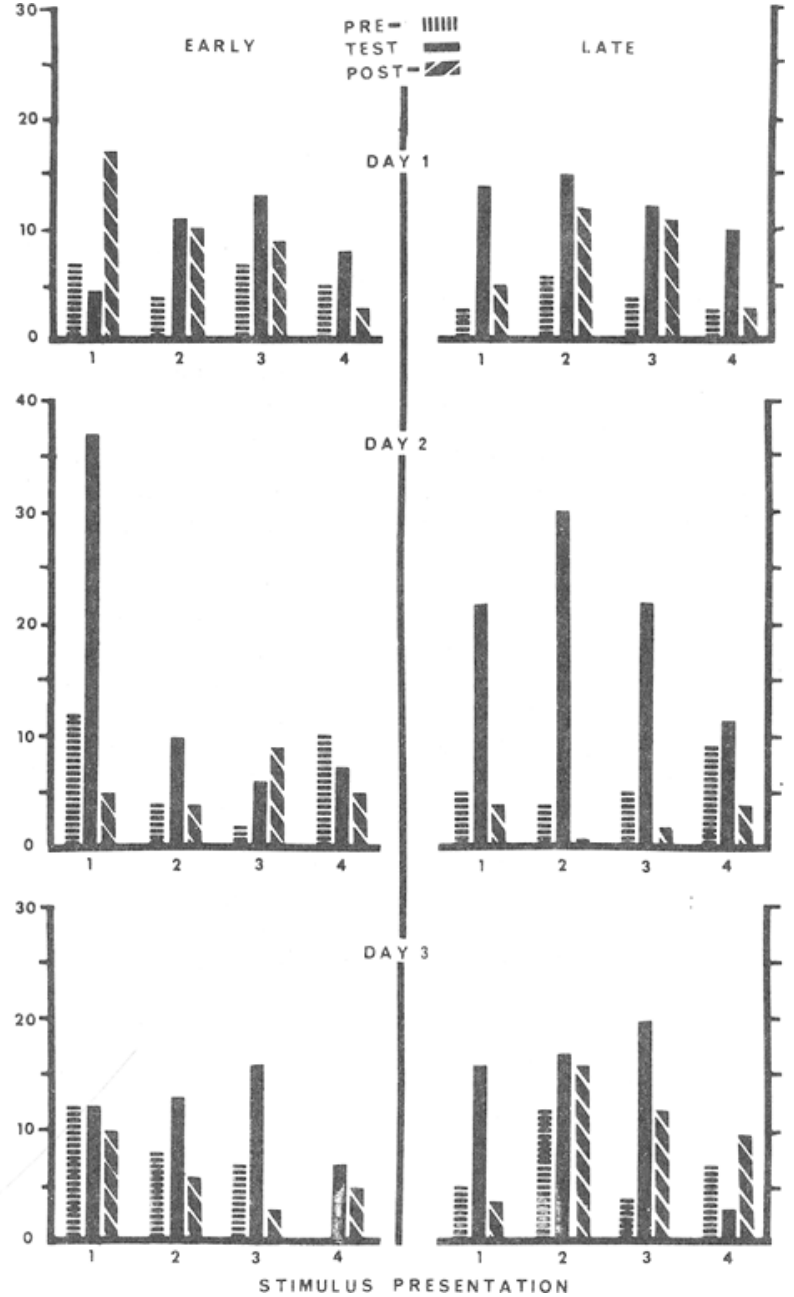

Fig. 1. Number of error responses emitted in the interval before, during, and after the four presentations of the novel stimulus either early or late on the 3 test days.

The results were analyzed using a four-factor analysis of variance (Winer, 1962). The major effect of presenting the potentially disinhibiting stimulus was assessed using the number of error responses before, during, and after the stimulus presentation. This effect was found to be statistically significant $(\mathrm{F}=14.26, \quad \mathrm{df}=2 / 32$, p <.01). In both the "early" and the "late" stimulus presentation groups, evidence of disinhibition was found in that there were more error responses during the periods when the novel auditory stimulus was presented than there were in the periods either before or after the stimulus presentation. Although we observed more error responses in the group stimulated late in the DRL interval, the effect of presenting the stimulus "early" or "late" was found to be statistically nonsignificant $(F<1, \quad d f=1 / 16)$. There was a significant decline in the number of error responses over the four stimulus presentations within any one test session $(F=3.12, \mathrm{df}=3 / 48$, $p<.05)$. In addition, the analysis of variance yielded a significant interaction between test days and the pre-, test, and postinterval factor.

\section{DISCUSSION}

Criterion performance on a DRL specified time interval. The present results show that this inhibition of leverpressing can be overcome or disinhibited by the presentation of a novel auditory stimulus during the DRL interval. When the novel stimulus was presented, an increase was observed in the number of leverpressing responses before the end of the specified interval, and this effect was found whether the stimulus was presented "early" or "late" in the interval. Evidence of weakening of the disinhibitory effect of the stimulus was also found in the decrease in the schedule of reinforcement requires inhibition of leverpressing during the number of error responses over the four stimulus presentations within each of the test sessions.

Regardless of the decline in the disinhibiting effect of the stimulus with added exposure, the magnitude of the differences attributable to pre-, test, and postinterval conditions depends on the particular day $(1,2$, or $3)$ on which the measure was taken. On Day 2 the disinhibiting effect of the test stimulus was greatest. Also on Day 2 the "recovery" during the posttest interval of the inhibition necessary for good DRL performance was most marked. The evidence for disinhibition obtained on the second day of testing seems the most unambiguous. It is postulated that on Day 1 the disinhibition effect is confounded with the S's orienting responses to the novel stimulus. By Day 3 the effect is comparatively reduced again by adaptation to the previously "novel" stimulus.

Two explanations of criterion performance on a DRL schedule have been proposed and each one in turn leads to a different interpretation of the results reported in this paper. Laties et al (1965) observed the spontaneous development of mediating behavior in a rat working on a DRL schedule. Laties et al (1969) further observed that such collateral mediating behaviors as wood nibbling facilitated the acquisition of stable DRL behavior. These results suggest that inhibition of leverpressing during the DRL interval is based upon the establishment of some mediating response chain which acts to separate leverpresses, the terminal member of the chain, by the required interval. In accord with this type of explanation, the results of the present experiment indicate that the presentation of the novel stimulus disrupted whatever collateral behaviors had developed and elicited the terminal member of the chain, the leverpress.

A more traditional explanation of criterion DRL behavior is based upon the concept of inhibition and states that during the DRL interval the response of leverpressing is actively inhibited. When this ongoing process is disrupted by the presentation of the novel stimulus, the leverpress is emitted. The significant increase in the number of responses during the test interval, compared to the base pre- and postrates of the operant lend support to the latter interpretation of DRL behavior and the disinhibition phenomenon. If disinhibition is the disruption of an established response chain and the elicitation of the terminal member, it is not clear why Ss repeatedly break the chain. However, according to the traditional explanation, with the inhibition 
dissipated, a comparatively high rate of leverpressing can be expected in the test interval.

The results of this experiment can be regarded as showing a disinhibition effect in an operant conditioning situation with a DRL schedule. As such, these results are similar to those previously reported with FI schedules (Ferster \& Skinner, 1957; Hinrichs, 1968; Singh \& Wickens, 1968), and those obtained by Pavlov (1927) using delayed classical conditioning procedures.

BRADY, J. V. \& CONRAD, D. G. Some effects of brain stimulation on timing behavior. Journal of the Experimental Analysis of Behavior, 1960, 3,93-106.

BROWN, P. L., \& JENKINS, H, $M$ Conditioned inhibition and excitation in operant discrimination learning. Journal of Experimental Psychology, 1967, 75 . 255-266.

FERSTER, C. B., \& SKINNER, B. F. Schedules of reinforcement. New York: Appleton-Century-Crofts, 1957.

FL AN A G A N, B., \& WEBB, W. B Disinhibition and external inhibition in fixed interval operant conditioning.
Psychonomic Science, 1964, 1, 123-124. HARTLINE, H. R. Inhibition of activity of visual receptors by illuminating nearby retinal elements in the Limulus eye. Federation Proce edings, 1949, 8, 69.

HINRICHS, J. V. Disinhibition of delay in fixed-interval instrumental conditioning. Psychonomic Science, 1968, 12, 313-314. LATIES, V. G., WEISS, B., CLARK, R. L. \& REYNOLDS, M. D. Overt "mediating" behavior during temporally spaced responding. Journal of the Experimental Analy sis of Behavior, 1965, 8, 107-116.

LATIES, V. G., WEISS, B., \& WEISS, A. B. Further observations of overt "mediating" behavior and the discrimination of time. Journal of the Experimental Analysis of Behavior, 1969 , $12,43-57$.

PAVLOV I. P Conditioned reflexes (translated by G V. Anrep). London: Oxford University Press, 1927.

SINGH, D., \& WICKENS, D. D Disinhibition in instrumental conditioning. Journal of Comparative \& Physiological Psychology, 1968, 66, $557-559$.

STADDON, J. E. R. Inhibition and the operant. Journal of the Experimental Analysis of Behavior, 1969, 12, 481-487.

WINER, B. J. Statistical principles in experimental design. New York McGraw-Hill, 1962. under conditions of continuous viewing. These findings support the hypothesis that occipital EEG varies inversely with the "arousal" value of visual input.

The Ss were 30 undergraduate first-year students (19 men and 11 women) at the University of Exeter, with an average age of 18 years and 9 months.

\section{APPARATUS AND PROCEDURE}

The $S$ sat in an adjustable hairdresser's chair and looked through a window cut into a black barrier screen, such that his visual field was limited to the view of a test slide projected onto a screen $12 \mathrm{ft}$ away on the other side of the barrier screen. The S's height was adjusted until his angle of regard was at $90 \mathrm{deg}$ to a center point on the test slide. The area of the projected slide was $30 \times 30$ in. Apart from the illumination of the projector screen (when this was in use), the $\mathrm{S}$ sat in the dark. The $\mathrm{E}$ was able to monitor the projected slides through a masked spyhole from the adjoining experimental room.

Five conditions of visual stimulation were employed (1) eyes shut, (2) eyes open in the dark, (3) eyes open viewing a blank screen, (4) eyes open viewing a black circle ( 10 -in. radius) on a white background, and (5) eyes open viewing the circle with eight spokes radiating at 45 -deg intervals. There were 102 -min trials presented in two blocks of the five conditions, random within blocks, with the constraint that no two identical conditions were adjacent. Total experimental time was, therefore, $20 \mathrm{~min}$. Whenever the $\mathrm{S}$ was required to open or shut his eyes, the $E$ called the instruction to him through the spyhole. On other occasions, either the main supply to the projector was switched on or off or the slide was changed automatically, without comment.

Silver-silver/chloride loose pad electrodes were placed transoccipitally for bipolar recording (Cooper et al, 1969) with the neutral electrode on the left wrist. Interelectrode resistance was always below $6 \mathrm{k}$ ohms. The primary EEG was recorded on a San'ei polygraph, calibrated to give $24 \cdot \mathrm{mm}$ writeout (peak to trough) for $100 \mathrm{microV}$, time constant $0.3 \mathrm{sec}$. Continuous writeout of nine separate pure bandpass filters, each integrated for a 5-sec epoch, was provided by a San'ei low-frequency analyzer, giving a resolution of $40 \times 1 \mathrm{~mm}$ intervals. The filters were set at the following frequencies: $2.0-4.5 \mathrm{cps}, 4.5-6.5 \mathrm{cps}$, 6.5-7.5 cps, $7.5-8.5 \mathrm{cps}, \quad 8.5-9.5 \mathrm{cps}$, $9.5-10.5 \mathrm{cps}, \quad 10.5-11.5 \mathrm{cps}$, $11.5-14.5 \mathrm{cps}$, and $14.5-20.0 \mathrm{cps}$. These filters were flat-topped and
* This work was partially supported by a grant from the Medical Research Council (Ref. G. 969/185/C) extends the number of viewing conditions. Apart from sleep research, where extensive EEG samples are taken, psychological studies of the EEG invariably employ discrete EEG measures in conjunction with brief exposure to stimulation. The technique we employ involves relatively long-term time sampling 